\title{
Research on Packing and Cutting Optimization System of Two-dimensional Irregular Parts Oriented to Excess Material
}

\author{
Zhaoyun $\mathrm{Wu}^{1, \mathrm{a}, *}$, Yulan $\mathrm{Zhang}^{1, \mathrm{~b}}$, $\mathrm{Li} \mathrm{Li}^{2, \mathrm{c}}$, Lihui $\mathrm{Wu}^{1, \mathrm{~d}}$ and Nanbo Liu ${ }^{1, \mathrm{e}}$ \\ ${ }^{1}$ School of Mechanical \& Electrical Engineering, Henan University of Technology, Lianhua Street, \\ Zhengzhou, China \\ ${ }^{2}$ Automobile Engineering Department, Henan Polytechnic, Pingan Street, Zhengzhou, China \\ a wzhaoyun@163.com, ${ }^{\mathrm{b}}$ 525458949@qq.com, ${ }^{\mathrm{c}}$ cflw2006@163.com, ${ }^{\mathrm{d}}$ wulihui@haut.edu.cn, \\ e lnb@ haut.edu.cn \\ *corresponding author
}

Keywords: Packing Optimization, Cutting Optimization, Excess Material

\begin{abstract}
In view of the problems of the current packing methods, the development framework of the packing and cutting optimization system of two-dimensional irregular parts oriented to excess material was proposed. Two models of the hybrid architecture of $\mathrm{C} / \mathrm{S}$ and $\mathrm{B} / \mathrm{S}$ were analysed. The architecture of the packing and cutting optimization system was presented based on the hybrid architecture. The design pattern based on the 3-layers architecture was discussed: the data storage layer, the application logic layer and the user interaction layer. Some key technologies of the system were put forward, including the fast retrieval and matching method of excess material, the parts packing optimization method based on multiple excess materials, and the cutting path optimization method based on multiple excess materials. Based on these, the packing and cutting optimization system of two-dimensional irregular parts oriented to excess material was designed and developed by using VS.NET.
\end{abstract}

\section{Introduction}

Since the 1970s, the packing optimization problem has been gotten extensive attention by many research institutions and researchers in the world, different researchers put forward a variety of methods from different discipline angles. Many papers were published in the related academic conferences and journals, and the related monographs and software were also released. Currently, there is little research on packing and cutting optimization oriented to excess material.

It is inevitable that there will be remnants during long-term production process, at present most enterprises treat remnants as waste material. The price of waste material is just 1/6 purchasing cost, which causes great waste. Facing the rising price of raw material, in order to save material and reduce cost, the excess material is preferentially used. Therefore, it is necessary to research on packing and cutting optimization of two-dimensional irregular parts oriented to excess material. Aiming at the problem, the packing and cutting optimization system of two-dimensional irregular parts oriented to excess material is designed in the paper.

\section{The Hybrid Architecture of $\mathrm{C} / \mathrm{S}$ and $\mathrm{B} / \mathrm{S}$}

The architectures of $\mathrm{C} / \mathrm{S}$ and $\mathrm{B} / \mathrm{S}$ all have respective disadvantages, so they should be combined to develop the information system. At present, the hybrid architecture of $\mathrm{C} / \mathrm{S}$ and $\mathrm{B} / \mathrm{S}$ mainly includes two models [1-2]. (1) Different inside and outside model. In the inside of enterprise, the software is built by the architecture of $\mathrm{C} / \mathrm{S}$, and the users can directly access the database server through the LAN. In the outside of enterprise, the software is built by the architecture of $\mathrm{B} / \mathrm{S}$, and the users access the database server through the Web server and Internet. In this model, the external users can not directly access the database server, which can improve the security of the data. For the internal users, the response speed of system operation is faster. (2) Different browsing and 
modifying model. In this model, the software which needs to modify or delete the data is designed based on the architecture of $\mathrm{C} / \mathrm{S}$. The software which just needs to read and browse the data is designed based on the architecture of $\mathrm{B} / \mathrm{S}$. This model combines the advantages of the two kinds of architecture model. But the external users can directly connect to the database server, so the data of the enterprise will face greater risk [3]. Based on the above analysis, the first model was adopted in this paper.

\section{The Function Modules and Structure of System}

The packing and cutting optimization system of two-dimensional irregular parts oriented to excess material is designed based on the hybrid architecture of $\mathrm{C} / \mathrm{S}$ and $\mathrm{B} / \mathrm{S}$. In the inside of the enterprise, the applications are based on the architecture of $\mathrm{C} / \mathrm{S}$. The function modules mainly include: system management, user management, permission management, material management, part model management, excess material selection subsystem, packing subsystem, cutting path optimization subsystem etc. The client software of $\mathrm{C} / \mathrm{S}$ works in the enterprise internal LAN, and has powerful user interface and fast response speed, which can be helpful for the employees to finish the work better. In the outside of the enterprise, the applications are based on the architecture of $\mathrm{B} / \mathrm{S}$. The function modules mainly include: order query, online interactive, function display, statistical report, etc. The client browsers of $\mathrm{B} / \mathrm{S}$ work in the Internet. They send request to the Web server by HTTP protocol, and then obtain response. This mode can effectively ensure the safety of the enterprise database. The hybrid architecture of the packing and cutting optimization system of two-dimensional irregular parts is shown in figure 1.

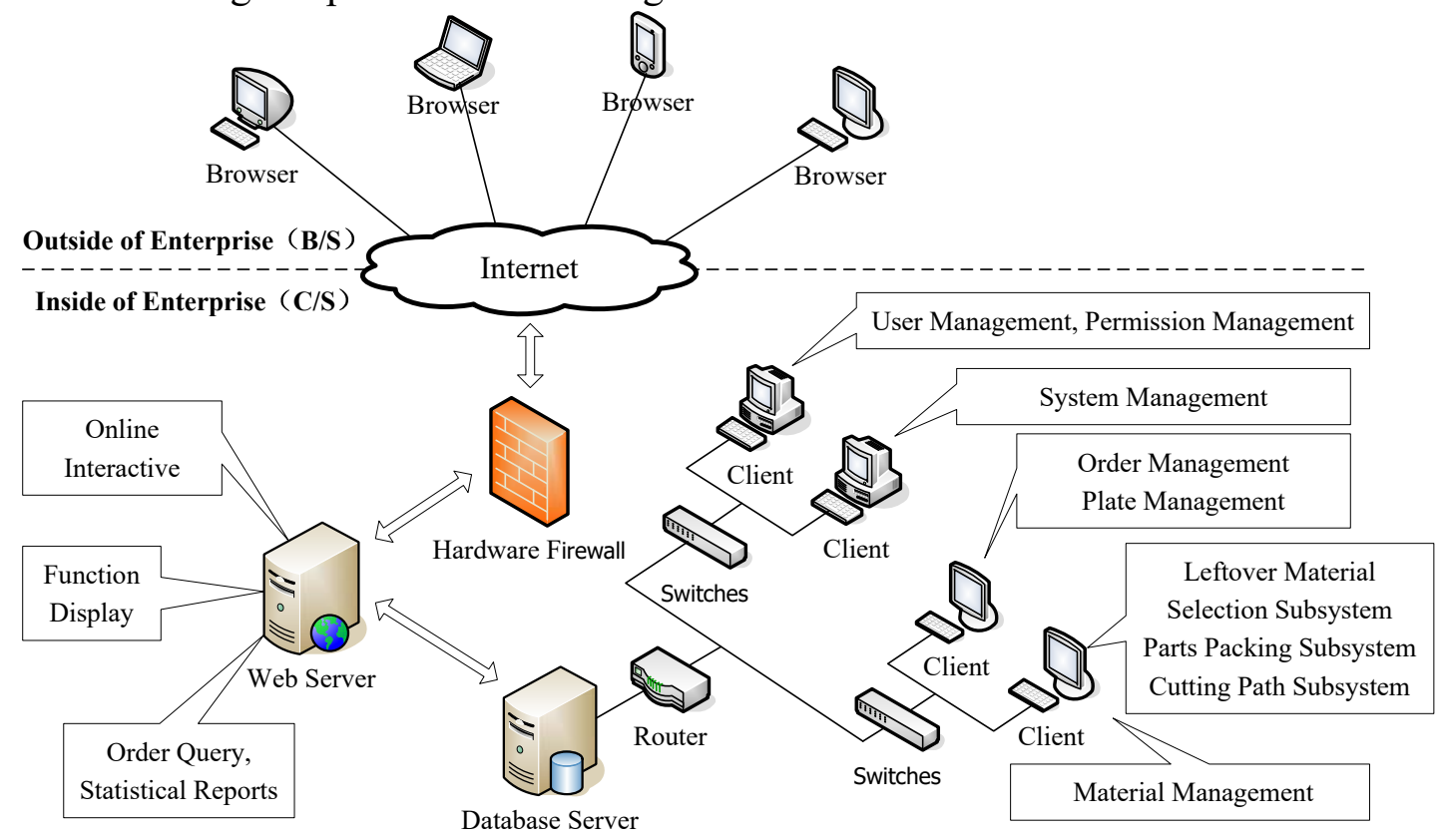

Figure 1 The structure of the hybrid architecture of system.

\section{System Design Model Based on the Three Layer Architecture}

In order to integrate the business of the enterprises better, the SOA architecture is taken as the design principle of the packing and cutting optimization system. Some systems are integrated into the platform, including the excess material selection subsystem, the packing optimization subsystem of parts, the cutting path optimization subsystem, etc. The overall structure of the system is divided into 3 layers: the data storage layer, the application logic layer and the user interaction layer, as shown in figure 2.

(1) Data storage layer. This layer is the basic data storage layer of the platform. The database management systems (SQL Server, Oracle, etc.), the XML technology and the file storage system are adopted in the platform. The databases mainly includes the basic data DB, the excess material 
$\mathrm{DB}$, the part model $\mathrm{DB}$ and the basic material DB, etc.

(2) Application logic layer. This layer is the core layer of the platform. The three business systems are integrated into this layer, involving the system management, the excess material selection and match, packing and cutting path optimization of parts, etc. The main goal of this layer is to realize the packing and cutting path optimization of two-dimensional irregular parts.

(3) User interaction layer. The interaction functions are realized in this layer, mainly including information retrieval, parameter input, remote access, packing pattern browse, result output, etc.

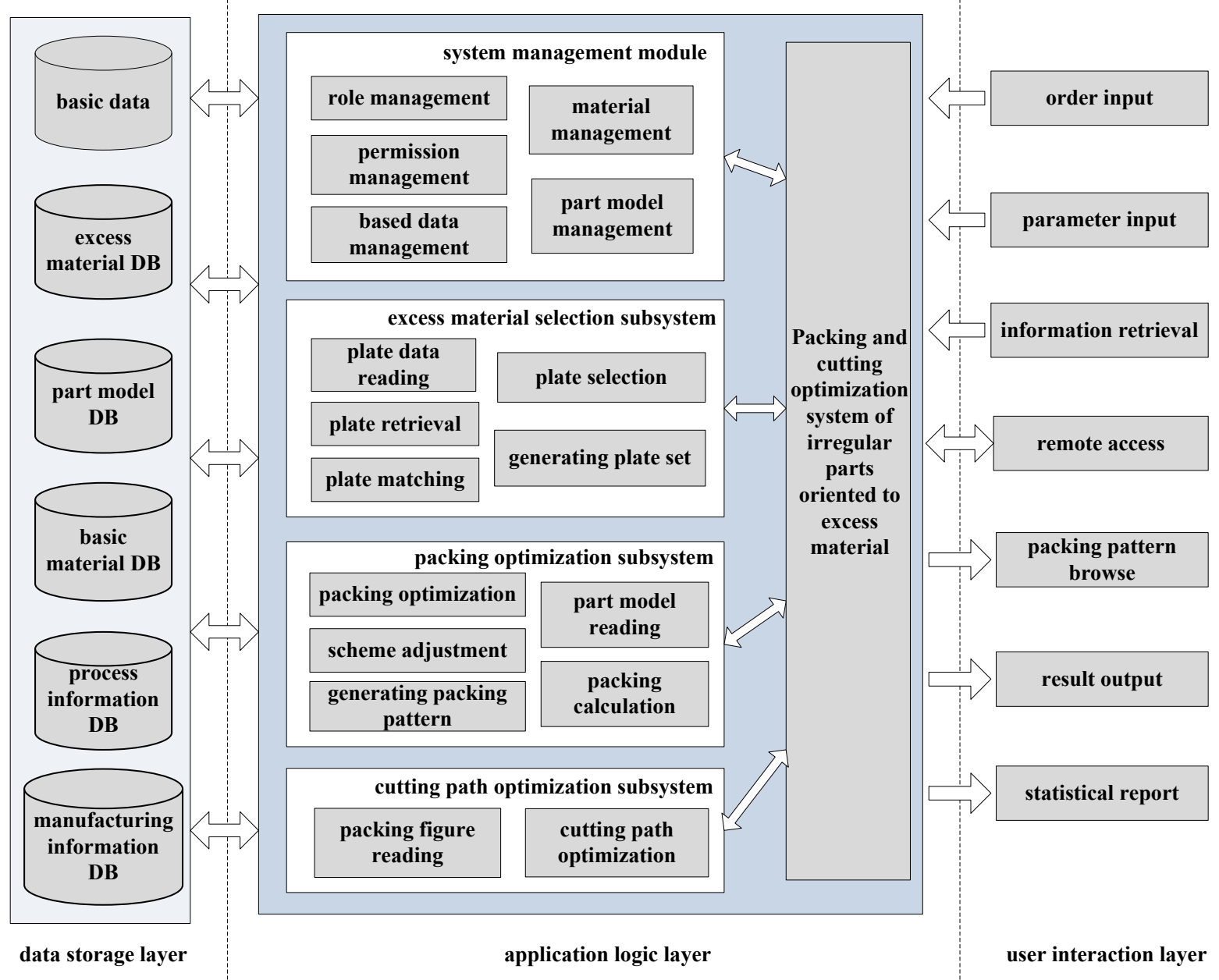

Figure 2 The platform structure based on the three layer architecture.

\section{Key Technologies of the System}

\subsection{The Fast Retrieval and Matching Method of Excess Material}

According to the shape characteristics of excess material, its characteristic parameters, mainly including the area of excess material, the coverage rate of enveloping rectangle, the approximate ratio between long and short axes, etc. are established. Taking the Holographic modeling principle as the theoretical basis, the multi view application model of excess material is set up. According to the hierarchical relationships of remnant characteristic parameters, the extended CAD holographic modeling is built. By adopting the method of correlation analysis and fuzzy analysis method, combining with the information retrieval principle of holographic model, the primary selection of remnants is done.

Using the least surrounding boxes algorithm of polygon, the least surrounding box for each unpacked part is generated. The maximum inscribed rectangle algorithm of polygon is adopted to calculate the maximum inscribed rectangle for each excess material. Then, the inclusion relation test between the two is processed to form the excess material set. 


\subsection{The Packing Optimization Method Based on Multiple Excess Materials}

Combining multi-objective coupling optimization theory with the method of mathematical analysis, taking the minimum plate area as the optimization goal, the packing optimization mathematical model of parts oriented to multiple excess materials is set up. According to the optimization mathematical model, using the basic principle of ant colony algorithm, the heuristic rules and taboo constraints of selecting excess material and the pheromone matrix of matching excess material and parts are established. Calling the placement method of parts, the synthesis algorithm of polygon is adopted to update the outline of the packing space. The ant colony optimization algorithm oriented to multiple excess materials is established to optimize the packing scheme of parts.

\subsection{The Cutting Path Optimization Method Based on Multiple Excess Materials}

Combining the group aggregation model of excess materials with the hierarchical constraint model of parts, the shortest idle travel of laser head is considered as the optimization goal. The mathematical model of cutting path optimization based on multi constraints is established [4]. After determining the cutting starting point of each part, the cutting path optimization problem can be transformed into the classical TSP problem. According to the mathematical model, using the basic principle of ant colony algorithm, the constraint rules of choice node by ants is set up. The multiconstraints ant colony optimization algorithm oriented to multiple excess materials is established to optimize the cutting path scheme of parts [5-6].

\section{Development and Implementation of the System}

Based on the analysis of the system architecture and key technologies, the packing and cutting optimization system of two-dimensional irregular parts oriented to excess material is designed and developed. Taking Visual Studio 2012 as development environment, using C\# as development language, SQL Server 2010 is used to establish the system database, the core algorithm of the module is completed in Matlab, and the parameter communication is finished by the hybrid programming technologies of Matlab and $\mathrm{C}$ \#. The secondary development of AutoCAD is done by $\mathrm{C \#}$ to realize graphics data modeling of excess materials and parts. Through AutoCAD parametric drawing technology, the packing graph and cutting path graph are drawn.

\section{Conclusion}

The packing and cutting optimization system of two-dimensional irregular parts oriented to excess material is established in the paper. The system architecture and design pattern are analyzed, and the key technologies of the system are put forward. The packing and cutting optimization is the core of the system. How to further improve the performance and operation speed needs to be further researched.

\section{Acknowledgements}

This work was supported by the National Nature Science Foundation of China (Grant No. 51405133), the Province Science and Technology Research Projects of Henan (Grant No. 172102210213), and the Fundamental Research Funds for the Henan Provincial Colleges and Universities in Henan University of Technology (Grant No. 2016RCJH02).

\section{References}

[1] Yang, J.W., Xue, Y.Q. and Liu, Z.P. (2006) Design of System Based on C/S and B/S Hybrid Software Architecture. Journal of Hebei University (Natural Science Edition), 26, 315-318,323.

[2] Liu, X.J., Liu, Y.H. and Wang, D. (2004) Implementation of Management System with Longitudinal Synthesized Structure Based on B/S and C/S Modes. Journal of Jilin University 
(Engineering and Technology Edition), 34, 146-149.

[3] Zhang, Y.S. and Chen, S.Q. (2002) C/S and B/S Mixed Software Architecture Model. Computer Engineering and Application, 38, 138-140.

[4] Liu, H.X., Wang, X. and Cai, L. (2004) Torch Path Optimization for NC Laser Cutting of Sheet Metal Part. Journal of CAD \& CG, 16, 660-665.

[5] Sun, H.P., Li, J. and Guo, W.G. (2008) Application of Genetic Algorithm on Optimization of High Power Beam Cutting Paths. Transactions of the Chinese Society for Agricultural Machinery, 39, 158-160,165.

[6] Li, N.N., Chen, Z.W. and Chen, S.Z. (2010) Optimization of Laser Cutting Path Based on Local Search and Genetic Algorithm. Computer Engineering and Applications, 46, 234-236,239. 